\title{
Simulación de interacción fluido-estructura en la red vascular utilizando el método de elementos de frontera (BEM)
}

\author{
John Bustamante Osorno ${ }^{1}$, Whady Felipe Florez E ${ }^{1}$, Andrés Felipe Hernández ${ }^{1, \psi}$ \\ ${ }^{1}$ Universidad Pontificia Bolivariana
}

Recibido 11 de enero de 2019. Aceptado 18 de mayo de 2019

Resumen-El principal objetivo de este trabajo fue desarrollar un modelo tridimensional, que permita el estudio de condiciones fluidodinámicas en un vaso sanguíneo, considerando el comportamiento pulsátil, mediante métodos numéricos de formulación integral y funciones de interpolación.

La metodología utilizada fue acoplar a un programa de elementos de frontera existente en el grupo de investigación, la entrada de la función pulsante que representa el comportamiento sanguíneo, como la ecuación a solucionar es la ecuación de Navier Stokes, se realizó el acople del término convectivo utilizando el método de funciones de base radial y para el avance temporal se implementó el esquema de Dual reciprocity method. Para la pared arterial, se calcularon los esfuerzos, se solucionó la matriz de esfuerzo deformación y finalmente se soluciona el esquema de anillos de presión para cada iteración.

En los resultados se desarrolló una geometría en el software GID con una relación de longitud a diámetro 4 a 1, se utilizó un número de Reynolds de 1061 y como condiciones de frontera se pusieron una condición de velocidad pulsante a la entrada y una condición de tracción a la salida.

Los resultados mostraron el desarrollo del perfil parabólico y los desplazamientos de pared que obedecen a los reportados en literatura $(10 \%)$.

La principal conclusión es que el uso de los métodos combinados, elementos de frontera y anillos de presión, sirven para simular la interacción entre los dos medios, respetando la homeóstasis del sistema biológico original. Además, se logró alcanzar un número de Reynolds de $1061 .$.

Palabras clave-Desplazamientos, Interacción fluido estructura, Método de elementos de frontera, Red vascular. 


\title{
Simulation Of Fluid-Structure Interaction In The Vascular Network Using The Border Element Method (BEM)
}

\begin{abstract}
The main objective of this work was to develop a three-dimensional model, which allows the study of fluid dynamic conditions in a blood vessel, considering pulsatile behavior, using numerical methods of integral formulation and interpolation functions.

The methodology used was to connect to an existing boundary elements program in the research group, the input of the pulsating function that represents blood behaviour, as the equation to be solved is the Navier Stokes equation, the convective term was coupled using the radial basis functions method and for the temporary advance the Dual reciprocity method scheme was implemented. For the arterial wall, the stresses were calculated, the strain stress matrix was solved and finally the pressure ring scheme for each iteration was solved.

In the results a geometry was developed in the GID software with a ratio of length to diameter 4 to 1 , a Reynolds number of 1061 was used and as boundary conditions, a pulsating speed condition was introduced at the entrance and a traction condition was applied in the output.

The results showed the development of the parabolic profile and the wall displacements that obey the reported in literature (10\%).

The main conclusion is that the use of the combined methods, boundary elements and pressure rings, serve to simulate the interaction between the two different media, respecting the homeostasis of the original biological system. In addition, a Reynolds number of 1061 was achieved..
\end{abstract}

Keywords - Gait rehabilitation, Lower limb exoskeleton, Superficial electromyography sEMG, User interface.

\section{SiMULAÇÃO DA INTERAÇÃO FLUIDO-ESTRUTURA NA REDE VASCULAR USANDO O MÉTODO DOS ELEMENTOS DE BORDA (BEM)}

\begin{abstract}
Resumo - O principal objetivo deste trabalho foi desenvolver um modelo tridimensional, que permita o estudo de condições dinâmicas de fluidos em um vaso sanguíneo, considerando o comportamento pulsátil, utilizando métodos numéricos de formulação integral e funções de interpolação.

A metodologia utilizada foi conectar-se a um programa de elementos de fronteira existente no grupo de pesquisa, a entrada da função pulsante que representa o comportamento do sangue, como a equação a ser resolvida é a equação de Navier Stokes, o termo convectivo foi acoplado usando as funções de base radial para o avanço temporário, foi implementado o esquema do método de reciprocidade dupla. Para a parede arterial, as tensões foram calculadas, a matriz de tensão de tensão foi resolvida e, finalmente, o esquema de anéis de pressão para cada iteração foi resolvido.
\end{abstract}

Nos resultados, uma geometria foi desenvolvida no software GID com uma razão entre comprimento e diâmetro de 4 para 1 , foi usado um número de Reynolds de 1061 e, como condições de contorno, uma condição de velocidade de pulsação foi introduzida na entrada e uma condição de tração foi aplicada em a saída.

Os resultados mostraram o desenvolvimento do perfil parabólico e os deslocamentos da parede que obedecem aos relatados na literatura $(10 \%)$.

A principal conclusão é que o uso dos métodos combinados, elementos de contorno e anéis de pressão, serve para simular a interação entre os dois meios diferentes, respeitando a homeostase do sistema biológico original. Além disso, foi alcançado um número de 1061 de Reynolds.

Palavras-chave - Reabilitação da marcha, Exoesqueleto do membro inferior, Eletromiografia superficial sEMG, Interface do usuário.

\section{INTRODUCCIÓN}

$\mathrm{M}$ últiples procesos fisiológicos de los organismos vivos pueden ser simulados y representados mediante el uso de modelos matemáticos que describen los fenómenos físicos y biológicos que ocurren en los mismos o en la interacción existente con el medio que los rodea. La interacción entre un tramo arterial y el fluido sanguíneo es un fenómeno compuesto por dos medios continuos de distinta constitución, que mediante una interfaz acoplan su comportamiento [1]. Se presenta una relación recíproca entre ambos medios, siendo posible al medir variables físicas, conocer lesiones locales derivadas de la variación anormal de cualquiera de los parámetros presentes en la interacción [2]. Por ser las enfermedades vasculares responsables de una alta tasa de morbimortalidad, los investigadores se han enfocado en el desarrollo de nuevas técnicas que permitan estudiar y entender el comportamiento y la aparición de estas patologías. Cuando se presenta deterioro de un vaso sanguíneo por una lesión 
severa que perturbe las condiciones normales de flujo, es necesario realizar un implante vascular. Estas lesiones pueden ser producidas por enfermedades congénitas, trauma y alteraciones degenerativas, las cuales conllevan alteraciones circulatorias importantes, que determinan incluso falla en el bombeo sanguíneo, insuficiencia cardiaca congestiva y muerte del paciente; otras sólo producen síntomas ocasionales relacionados con el incremento en la demanda de bombeo, como ocurre durante la actividad física $[3,4]$. Muchas de las enfermedades vasculares pueden intervenirse mediante cirugía, con implantes biológicos o artificiales, los cuales permitirán restablecer la funcionalidad del sistema circulatorio. Por sus propiedades, los injertos biológicos procedentes de donantes cadavéricos (homoinjertos) son los más apropiados para cirugías reconstructivas, pero infortunadamente son difíciles de adquirir ya que se obtienen de donantes, con la restricción de las dimensiones y geometría de la estructura nativa a reparar [4].

El uso de simulaciones numéricas, unido a los avances en equipos de imágenes médicas y la alta velocidad de procesamiento con que cuentan los equipos computacionales actuales, han permitido la introducción de alternativas diferentes a los procedimientos utilizados tradicionalmente para detección de enfermedades vasculares que permiten al especialista tener una información completa sin necesidad de recurrir a técnicas invasivas [5, 6]. La aplicación de técnicas de biomecánica computacional al sistema vascular humano implica cuatro dificultades:

- Complejidad en la geometría del árbol vascular.

- Deformación en las fronteras entre paredes arteriales y el lumen vascular.

- Variaciones en el flujo sanguíneo debido a las condiciones de pulsatilidad.

- Manejo de términos no lineales en las ecuaciones diferenciales constitutivas de la sangre.

Estos elementos no son independientes. Es por esto que los métodos de simulación computacional requieren resolver modelos de diversos fenómenos físicos que actúan sobre ellos (acoplados) en diversas dimensiones tanto espaciales como temporales [7]. En tal sentido, el reto de la simulación numérica y pruebas experimentales es abordar el fluido sanguíneo real en arterias, relacionados con la reología o comportamiento no-Newtoniano de la sangre, la disposición característica de la pared arterial, la composición de la pared en capas, la determinación del fluido pulsátil entrante, el proceso del transporte de masa y la transición a fluidos turbulentos inestables, entre otros [8]. Permitiendo evaluar el comportamiento de la arteria ante variaciones de frecuencia, volumen latido y presión en un flujo pulsante $[4,9,10]$. La simulación computa- cional de fluidos en arterias patológicas representadas con geometrías reales derivadas de imágenes por resonancia magnética está ganando terreno ya que es una herramienta que permite entender y predecir una falla circulatoria. Esta simulación obvia la realización de estudios en modelos reales, ya que si se considera la toma de medidas in vivo del campo de velocidades en una arteria, se facilita el estudio al evitar efectos secundarios cuando se realiza una reconstrucción detallada de la geometría de la arteria a estudiar. Realizar una modelización con geometrías reales y utilizando diferentes modelos numéricos del fluido arterial, puede proporcionar información a los investigadores de cómo modificar o perturbar las condiciones de flujo que permitan conocer o predecir el comportamiento de distintas lesiones que ocurran en el árbol vascular [11].

Las simulaciones del sistema vascular presentan aún algunas limitantes, entre las cuales se encuentran:

Con respecto a la pulsatilidad de la sangre, debido a esto no se puede despreciar el tiempo considerando una solución en estado estable, ya que es función de la posición espacial

Considerando la interacción mecánica de la pared con el fluido. Este aspecto es relevante para vasos relativamente largos. En la aorta por ejemplo el radio puede variar en un rango de un $5 \%$ a un $10 \%$ entre la sístole y diástole. Esta variación afecta el fluido [5, 10, 22].

No existe un modelo en la interacción solido fluido, que considere la aparición de flujos secundarios y fenómenos locales como vórtices en el fluido sanguíneo $[12,13]$.

Dichas limitaciones presentadas por las diversas simplificaciones que se realizan al momento de simular sistemas biológicos pueden derivar en herramientas de estudio que no provean la suficiente versatilidad para explorar el comportamiento del sistema. Se observa como el manejo de la condición de flujo pulsátil existente en el sistema cardiovascular representa un punto fundamental al momento de abordar la investigación, ésta es responsable de las oscilaciones en las presiones y velocidades [14]. Así mismo el comportamiento del flujo depende de la elasticidad de las paredes y de la reología sanguínea, donde la elasticidad de las arterias funciona como un regulador de flujo [12]. El planteamiento y solución de modelos matemáticos del flujo sanguíneo en los que se tiene en cuenta la interacción con la pared arterial flexible es muy reciente $[15,16]$. Esto es debido a que los modelos son lo suficientemente complejos para tener que usar métodos numéricos híbridos para resolverlos [12]. Pretendiendo llenar un vacío en el estudio computacional del comportamiento de implantes vasculares se pretende realizar un acercamiento al problema de la interacción sanguínea con unas condiciones de frontera entregadas por los dos 
medios diferentes de tramo arterial (tramo sano y tramo con injerto) con un enfoque diferente tanto en el método numérico para resolver las ecuaciones constitutivas del modelo que representa la sangre, como en la interacción ocurrida con la estructura. La importancia de esta investigación radica en el uso de métodos numéricos de formulación integral y técnicas de simulación, para representar el comportamiento hemodinámico tras el implante de un injerto vascular, considerando el cambio de medio existente y la condición de pulsatilidad presentada, donde se observan las variaciones en los parámetros presentes en la interacción, siendo éste un problema biológico de dominios acoplados. Entre los métodos numéricos actuales, se encuentra los elementos de frontera, el cual por ser un método integral presenta ventajas computacionales sobre los tradicionales. También con el método de funciones de base radial es posible solucionar las no linealidades presentes en la ecuación de Navier-Stokes la cual rige el comportamiento del fluido sanguíneo, y con la solución entregada es posible evaluar el flujo en el sistema vascular sometido a unas características dadas por la frontera con la pared arterial [17]. Actualmente, las investigaciones se han centrado en el desarrollo de modelos unidimensionales que resuelven el acoplamiento fluido-pared arterial. Se asume simetría axial de todos los vasos y se utiliza para simular arterias principales con sus ramificaciones. El problema es que no entrega valores de campos tridimensionales ni de tensiones y velocidades $[18,12]$. Otros métodos iterativos, utilizan una solución para el fluido y otra para el sólido, en donde la convergencia de cada paso de tiempo se realiza mediante el método de punto fijo [19, 20, 21]. Cuando se trata de geometrías complejas se aplica, por ejemplo, el método de Lattice Boltzmann $[12,19]$.

\section{Materiales y MÉTodos}

Desde el punto de vista de la reología, los fluidos más sencillos son los Newtonianos, llamados así porque su comportamiento sigue la ley de Newton: El esfuerzo de corte es proporcional al gradiente de velocidad o velocidad de corte [28]

$$
\begin{gathered}
\rho \frac{\partial u_{i}}{\partial t}+u_{j} \frac{\left(\rho \partial u_{i}\right)}{\partial x_{j}}=-\frac{\partial p}{\partial x_{i}}+\mu \frac{\partial^{2} u_{i}}{\partial x_{j} \partial x_{i}} \\
\frac{\partial u_{i}}{\partial x_{i}}=0
\end{gathered}
$$

donde $u$ es la velocidad, $p$ es la presión, $\mu$ es la viscosidad y $\rho$ es la densidad en la ecuación de Navier Stokes (1).

El método de elementos de frontera es una herramienta numérica para solucionar ecuaciones integrales en la frontera $\Omega$ de superficie $S$ basado en un procedimiento de discretización.

Parte de la ecuación integral [24]:

$$
\begin{gathered}
u(x)=\int_{S} K_{i j}(x, y) u_{j}(y) d s_{y} \\
-\int_{S} u_{i}^{k}(x, y) \sigma_{i k}(y) n_{k} d s_{y} \\
+\int_{\Omega} u_{i}^{k}(x, y) g_{i}(y) d \Omega_{y}
\end{gathered}
$$

Con las soluciones fundamentales:

$$
\begin{gathered}
u_{i}^{k}(x, y)=-\frac{1}{8 \pi \mu}\left(\frac{\delta_{i j}}{r}+\frac{\left(x_{i}-y_{i}\right)\left(x_{k}-y_{k}\right)}{r^{3}}\right) \\
K_{i j}(x, y)=\sigma_{i k}\left(\vec{u}^{k}(x, y)\right) n_{k}(y)= \\
-\frac{3}{4 \pi}\left(\frac{\left(x_{i}-y_{i}\right)\left(x_{j}-y_{j}\right)\left(x_{k}-y_{k}\right) n_{k}(y)}{r^{5}}\right)
\end{gathered}
$$

Es un método que se aplica cuando se puede calcular las funciones de Green [29]. Con esto, es posible resolver ecuaciones diferenciales homogéneas sujetas a condiciones de frontera [30]. La estabilidad del método depende de su formulación integral; ya que al no ser un método derivativo. La integral tiene mejor estabilidad que la derivada por su tendencia a cero y no a infinito [31].

Se busca discretizar la ecuación (1) para llegar a una solución matricial de tipo

$$
G t+H u=0
$$

Donde $G$ y $H$ son las matrices de velocidades y tracciones con los coeficientes de la solución fundamental, y $u, t$ son vectores de las velocidades nodales $\mathrm{y}$ tracciones respectivamente.

Como la ecuación (1) tiene un término convectivo, se debe utilizar el método de reciprocidad dual multidominio para dicha discretización, la cual parte de la ecuación:

$$
u_{i}^{(l)}(x)=\sum_{m=1}^{n t} f\left(x, y^{m}\right)\left(\beta_{i}^{m}\right)^{(l)}
$$

Se busca conocer el valor de la velocidad en cada uno de los nodos del dominio:

$$
\left\{u_{i}^{(l)}\right\}=[F]\left\{\beta_{i}^{(l)}\right\}
$$


Para el cálculo de la derivada parcial, utilizando DRM, se derivan la matriz de funciones y se multiplica por los coeficientes

$$
\begin{gathered}
\frac{\partial u_{i}^{(l)}(x)}{\partial x_{j}}=\sum_{m=1}^{n t} \frac{\partial}{\partial x_{j}}\left[f\left(x, y^{m}\right)\right]\left(\beta_{i}^{m}\right)^{(l)} \\
\left\{\frac{\partial u_{i}^{(l)}}{\partial x_{j}}\right\}=\left[\frac{\partial F}{\partial x_{j}}\right]\left\{\beta_{i}^{(l)}\right\} \\
\frac{\partial F}{\partial x_{j}}(k, m)=\frac{\partial}{\partial x_{j}}\left[f\left(y^{k}, y^{m}\right)\right], k, m=1 \ldots n t \\
\left\{\frac{\partial u_{i}^{(l)}}{\partial x_{j}}\right\}=\left[\frac{\partial F}{\partial x_{j}}\right][F]^{-1}\left\{u_{i}^{(l)}\right\}
\end{gathered}
$$

Utilizando el método de Kansa, y según el uso de funciones de base radial, el termino convectivo es la velocidad nodal, por la derivada de la matriz de funciones que multiplican la solución del sistema que son la inversa de la matriz de funciones por los valores nodales de la velocidad.

$$
\left\{g_{i}^{(l)}\right\}=\rho\left[U_{j}^{(l)}\right]\left[\frac{\partial F}{\partial x_{j}}\right][F]^{-1}\left\{u_{i}^{(l)}\right\}
$$

La ecuación (13) entraría a la solución general matricial.

En la ecuación (1), también se presenta un término de aceleración temporal, el cual debe ser discretizado mediante el método de diferencias finitas, con:

$$
\frac{\partial u_{i}}{\partial t}=\frac{\left(u_{i}^{m+1}-u_{i}^{m}\right)}{\Delta t}
$$

Cuando se tienen ambos términos (convectivo y derivada temporal) $(13,14)$, la función es:

$$
\begin{gathered}
g_{i}^{(l)}=\rho\left(\frac{\partial u_{i}}{\partial t}+u_{j} \frac{\partial u^{i}}{\partial x_{j}}\right)^{(l)} \cong \\
\rho\left\{\left[U_{j}\right]\left[\frac{\partial F}{\partial x_{j}}\right][F]^{-1}+\frac{1}{\Delta t}\right) \\
\left.\left\{u_{i}^{(l) m+1}\right\}-\frac{\left\{u_{i}^{(l) m}\right\}}{\Delta t}\right\}
\end{gathered}
$$

\section{Solución Numérica}

Cuando se tiene el sistema. $H$ y $G$ son matrices cuadradas, los coeficientes en los que están calculadas al integrar los productos $\hat{u} \mathrm{y} \hat{t}$ por la función de interpolación respectivamente sobre cada elemento de la frontera.

$$
G t+H u=(G \hat{t}-H \hat{u}) \alpha
$$

Con la evaluación de la función de interpolación en los puntos nodales

$$
\alpha^{m}=(F)^{-1} \rho \frac{\partial u_{i}\left(y^{m}\right)}{\partial t}
$$

Reemplazando (17) en (16) se obtiene

$$
G t=H u+S \dot{u}
$$

Donde el punto denota la derivada temporal y:

$$
S=F^{-1}(G \hat{t}-H \hat{u})
$$

Para el avance temporal se propone por simplicidad, un esquema de integración de dos niveles, con una aproximación lineal para cada paso de tiempo, utilizando el esquema de Crank-Nicholson.

$$
\begin{gathered}
u_{u}=\left(1-\theta_{u}\right) u_{i}^{m}+\theta_{u} u_{i}^{m+1} \\
u_{i}=\frac{1}{\Delta t}\left(u_{i}^{m+1}-u_{i}^{m}\right)
\end{gathered}
$$

con $\theta_{u}$ siendo un parámetro que posiciona el valor de $u_{i}$ entre $m$ y $m+1$, normalmente se le asigna un valor de 0.5 [28] y de ahí los valores de $u_{0}$ serán los valores iniciales del problema.

Cuando se conocen los valores de $u_{i}^{m}$ pasarán a ser los valores de $u_{i}^{m+1}$ hasta que la respuesta sea encontrada. [27]

Para la pared arterial y el implante, Si se tiene un recipiente de pared delgada $[32,33]$, es necesario conocer el valor de la presión normal. El método de elementos de frontera (BEM), entrega el valor de la tracción en la frontera del dominio.

$\mathrm{Si}$

$$
\text { Presión }=\frac{1}{3} \sum \sigma_{i i}
$$

Entonces

$$
\text { presión } n_{\text {normal }}=\text { presión }{ }^{*} n
$$


Con

$$
n=\frac{(x, y, x)}{\sqrt{x^{2}+y^{2}+z^{2}}} \cdot \hat{n}
$$

Ahi se realiza una partición del dominio por medio de anillos de presión, con presión radial uniforme, donde se toman una división de 20 anillos en todo el volumen:

$$
\begin{gathered}
\sigma_{1}=0 \\
\sigma_{2}=\frac{p^{*} R}{2 t h} \\
\Delta R=\frac{p^{*} R^{2}}{2 E^{*} t h}
\end{gathered}
$$

Donde $p$ presión, $\sigma_{1}$ esfuerzo longitudinal, $\sigma_{2}$ esfuerzo radial, $R$ radio del cilindro, $\Delta R$ desplazamiento radial, th grosor de la pared, $E$ módulo de Young. $\Delta R$ entrega el cambio del desplazamiento radial, por tanto, este valor cambia en la geometría inicial, para realizar una nueva iteración.

\section{Resultados}

Se desarrolló una geometría en el software Gid 9.0.4, con una relación de longitud a diámetro de 4 a 1 . Para replicar el fenómeno con un número de Reynolds que pudiera correr la simulación según el método escogido, se tomaron los valores de diámetro $1 \mathrm{~cm}$ y longitud $4 \mathrm{~cm}$, conservando la relación longitud a diámetro. La geometría está normalizada en diámetro, por tanto, en los siguientes cálculos se guardará dicha relación [26].

Cuando se construyó la malla se diseñaron las condiciones de frontera para la solución del problema, esto se realizó en un software diseñado en Fortran, el cual crea el archivo de entrada para el desarrollo de la simulación.

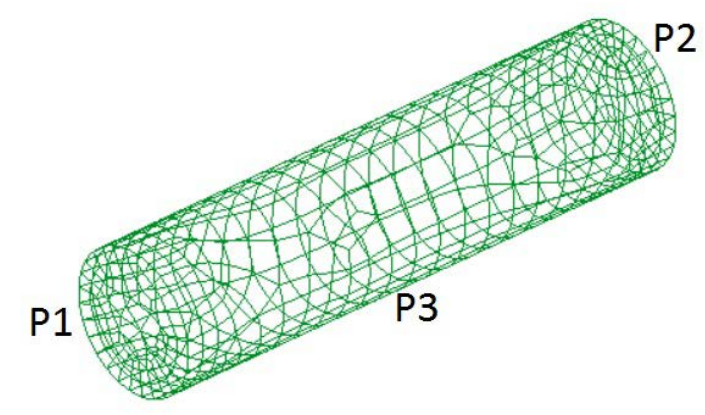

Fig.1. Gráfica donde se observan las caras y la superficie del vaso para definir las condiciones de frontera correspondientes al problema a simular.
Donde P1, P2, P3 corresponden a las caras de la geometría en las cuales se definen condiciones de velocidad de fluido $(\mathrm{Vx}, \mathrm{Vy}, \mathrm{Vz})$ o condiciones de Tracción (Tx, Ty, Tz). Según el problema estudiado, las condiciones de frontera que se deben definir son:

Tabla 1. Condiciones de frontera

\begin{tabular}{ccc}
\hline $\mathbf{P 1}$ & $\mathbf{P 3}$ & $\mathbf{P 2}$ \\
\hline $\mathrm{Vx}=$ función pulsante & $\mathrm{Vx}=0$ & $\mathrm{Tx}=0$ \\
$\mathrm{Vy}=0$ & $\mathrm{Vy}=0$ & $\mathrm{Vy}=0$ \\
$\mathrm{Vz}=0$ & $\mathrm{Vz}=0$ & $\mathrm{Tz}=0$ \\
\hline
\end{tabular}

La función pulsante, en la condición de frontera de entrada, emula o representa la condición fisiológica de apertura y cierre de las válvulas cardiacas, su representación se realiza matemáticamente desde una función senoidal, esta función depende del valor del caudal y de la posición nodal.

En cada paso de tiempo entrega el valor de la velocidad de los nodos de entrada del problema y está dada por la ecuación:

$$
U=\frac{2 Q}{\pi R^{4}}\left(R^{2}-r^{2}\right)\left(\frac{\sin \left(\frac{2 \pi t}{T}+1\right)}{2}\right)
$$

En donde $Q$ es el caudal máximo en el ciclo cardiaco, $R$ es el radio del vaso, $r$ es la variación del radio, $T$ es el periodo del ciclo cardiaco.

Con las condiciones de frontera establecidas, se encuentra la solución de perfiles de velocidad (Fig.2).

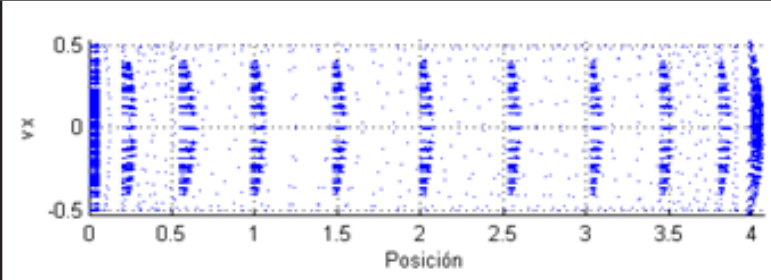

Fig.2. Perfiles de velocidad en una malla con 2630 nodos en la frontera y 576 nodos internos en dos dimensiones, la velocidad está dada en $\mathrm{cm} / \mathrm{s}$ y tanto el diámetro como la longitud están dadas en milímetros

La Fig 2, representa el perfil de velocidad del flujo sanguíneo visto en dos dimensiones. Se aprecia bien dicho flujo ya que como se mencionó anteriormente la precisión del método para representar cualquier fenómeno depende en gran medida de la calidad de la malla. El principal inconveniente de dicha malla es que, al pasar a un estado transitorio, genera problemas por falta de memoria. 
Se realizó un programa en Matlab, el cual muestra el comportamiento de la función pulsante en la simulación. Dicha función está representada por una señal senoidal desplazada en una unidad. Aunque se puede acotar dicha señal para trabajar solo el semiciclo positivo, diversos autores han utilizado señales parecidas a la forma escogida $[25,23,14,3,24]$.

Se desarrolló en Matlab, un algoritmo que muestra como seria el comportamiento analítico de la función pulsátil ecuación (28).

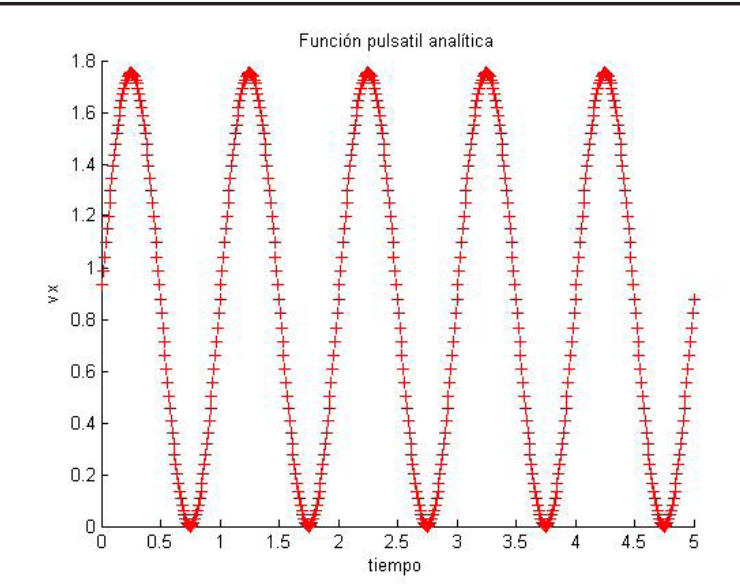

Fig.3. Función pulsátil analítica, desarrollada en un programa en Matlab, muestra cómo sería el comportamiento en un nodo que se encuentra en entrada del vaso, en el eje x se muestra el valor del tiempo en segundos, en el eje y se muestra la amplitud de la velocidad.

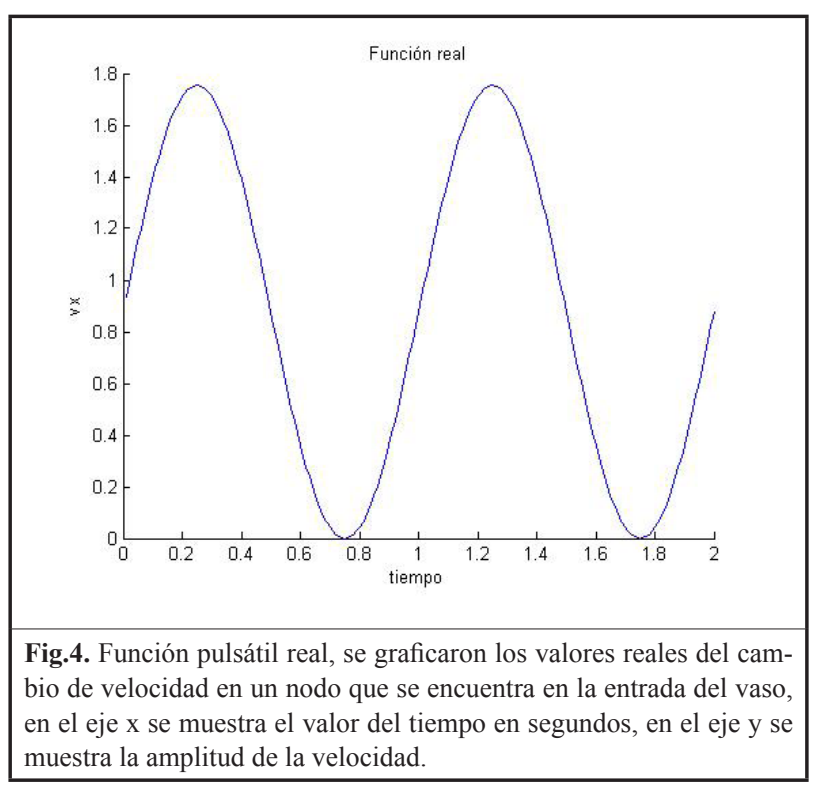

Como se tiene una representación de la función pulsante, se agrega al programa, se realiza el seguimiento de la función en un nodo que se encuentra en la frontera, el valor de dicha función se observa en la Fig.4.

Si se comparan los valores de la velocidad en los nodos que se encuentran a contra cara, se observa que a medida que esta aumenta a medida que la geometría se acerca a su valor real.

Cuando se corre una simulación con varios pasos de tiempo (1000), se observa como la velocidad aumenta y disminuye, siguiendo una función armónica y periódica, según se observa en la Fig.5.

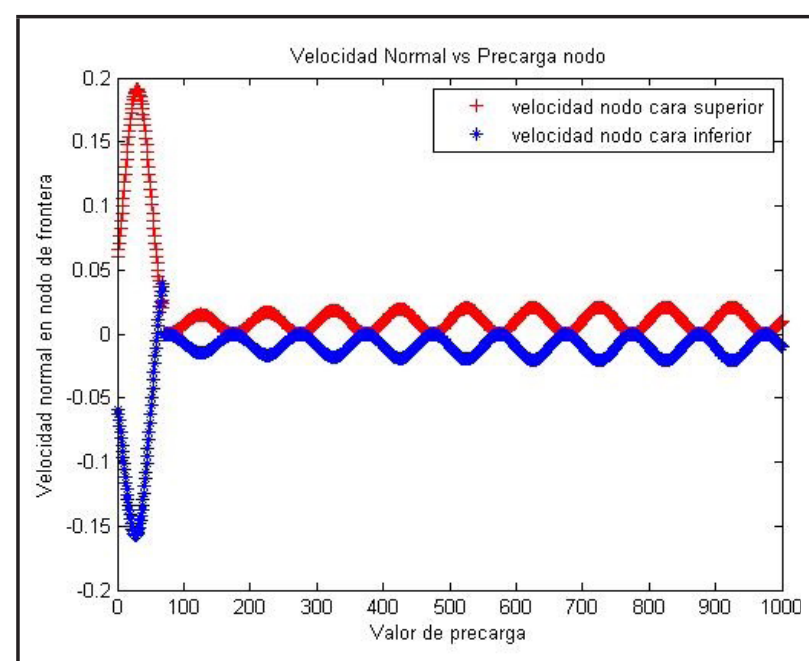

Fig.5. Velocidad en los nodos con un mayor número de iteraciones (1000).

La Fig.5, muestra la simetría que se presenta en la velocidad de dos nodos que se encuentran en caras opuestas de la geometría. Es importante ver que no existe asimetrías $\mathrm{y}$ tanto crestas como valles, presentan el mismo valor y comportamiento (con sentido opuesto).

Se realizó la comparación cuantitativa y cualitativa de la onda de presión arterial tomada de los libros de anatomía y la onda de presión presentada en este documento en $\mathrm{mmHg}$, se tomó además la literatura que reporta que el desplazamiento nodal no debe ser mayor al $10 \%$ [44]. Calculando el valor del desplazamiento nodal máximo, se tiene que equivale a $3,6 \%$, por lo tanto, se respeta lo reportado en la literatura. Esto se observa en la Fig. 6.

Luego de calcular los errores puntuales en cada nodo, se representa dicho error mediante una gráfica, donde el error máximo entre ambas gráficas es 3,14, lo cual se observa en la Fig. 7: 


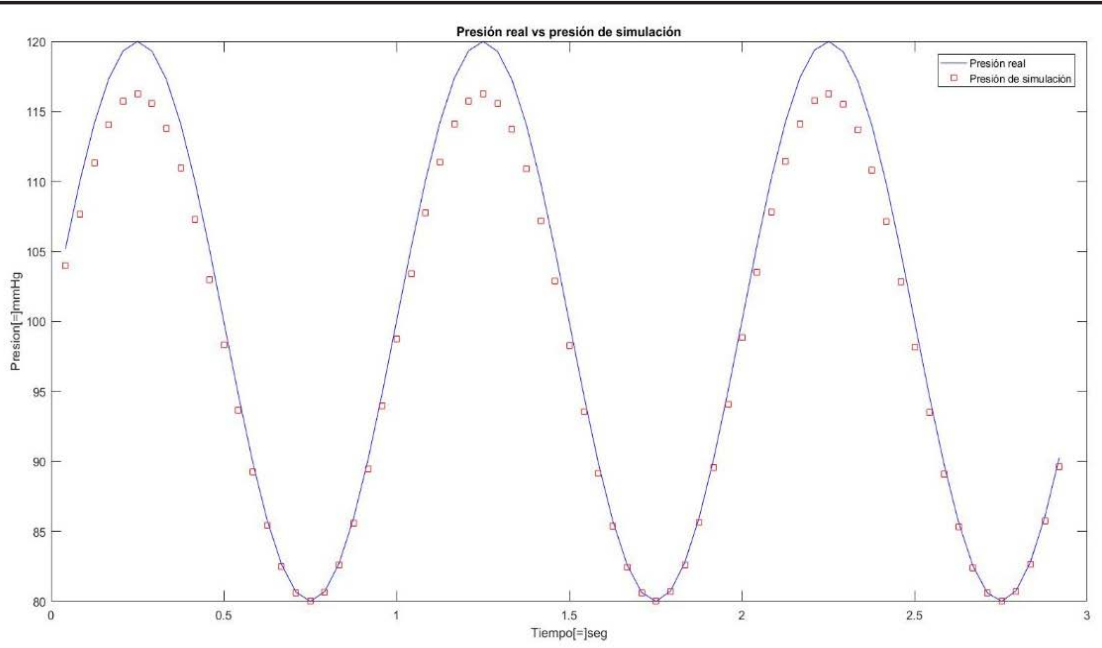

Fig.6. Comparación de la presión real representada como una sinusoide vs presión simulada.

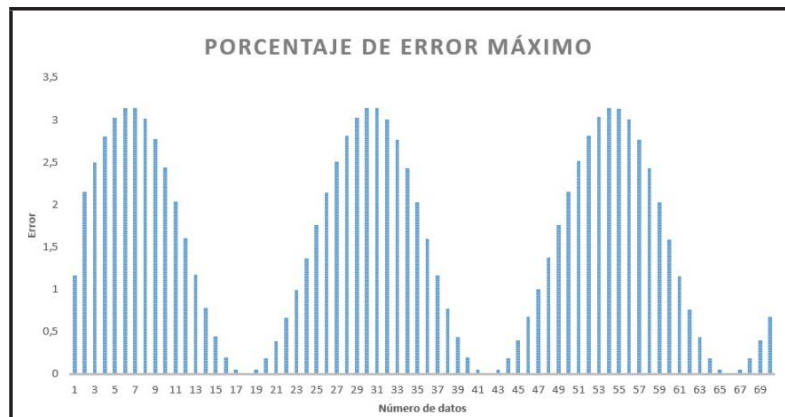

Fig.7. Porcentaje de error máximo entre presión real vs presión simulada

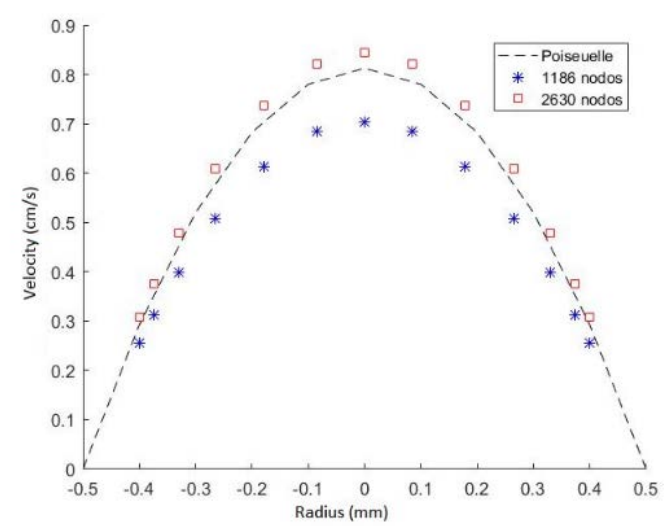

Fig.8. Gráfica comparativa entre la solución analítica y dos malas distintas.

Finalmente, para ver la veracidad de los resultados, se compara el modelo transitorio aumentando el número de nodos, de 1186 a 2030 con la solución analítica de Poiseuelle en la Fig. 8, esto demuestra que a medida que se aumenta la cantidad de nodos en la geometría, mejora la solución del problema respecto a la solución analítica, pero tiene un mayor consumo de recursos y de tiempo.

\section{Discusión}

El principal aporte de este artículo es el estudio de la interacción fluido estructura entre un tramo arterial, y la sangre; dicho estudio parte de las ecuaciones constitutivas matemáticas de Navier Stokes y las ecuaciones de Navier para elasticidad. Otro de los aportes es la programación de un método híbrido que combina la solución de la ecuación de Navier Stokes mediante el método de elementos de frontera, el manejo del término convectivo mediante las funciones de base radial multicuádricas con esquema upwind [35], y la aceleración convectiva mediante diferencias finitas.

Pocos autores han centrado su atención en desarrollo de códigos que permitan conocer la influencia de las variables fluidodinámicas y mecánicas en el comportamiento de un tramo arterial, actualmente las investigaciones se decantan por las condiciones de porosidad y la influencia geométrica, pero pocos consideran las condiciones de pulsatilidad, el cambio de medio, la onda de presión viajera y un vaso mayor como el presente artículo, es por eso que se tiene un gran punto de partida para el desarrollo de un esquema más robusto que permita tener una predicción a una mayor escala de tiempo.

\section{Conclusiones}

El método de elementos de frontera, junto con el método de reciprocidad dual multidominio y funciones de base radial, sirven para simular el comportamiento hemodinámico y permitieron desarrollar un modelo tridimensional que facilita el estudio de las condiciones fluidodinámicas en un vaso sanguíneo, en la entrada y fronteras, considerando el comportamiento pulsátil. 
Según la literatura, el desplazamiento en los vasos cuando se presenta la interacción fluido estructura es del 10 al 20\% [22] con respecto al diámetro del vaso, el modelo de anillos de presión; limita el sobre desplazamiento presentado en los nodos y cumple lo propuesto por la literatura.

El uso de los anillos de presión permite el acople en dos vías propuesto pues en el fluido se realiza el cálculo de la tracción, variable que entrega la presión normal sobre la pared en donde se encuentra finalmente los cambios en la geometría llevando al programa a realizar las posteriores iteraciones con este esquema.

El problema es tratado como una entidad monolítica y todas sus componentes avanzan simultáneamente en el cálculo. Este es el método de la descripción Arbitrary Lagrangian Eulerian (ALE), considerado según Felippa [34] el marco adecuado para los problemas con paredes deformables.

$\mathrm{Al}$ aumentar el valor del número de Young en el material estudiado, se presenta un mayor nivel de presión sobre este y el desplazamiento nodal disminuye, pero a su vez tiende a soluciones de pared rígida.

Cuando se realiza la comparación cuantitativa y cualitativa de la onda de presión entre el modelo y la realidad, se tiene que el porcentaje de error es $3 \%$, por tanto, el modelo sirve para representar el fenómeno, esto se toma considerando el comportamiento de la onda de presión como una onda sinusoide y tomando sus valores característicos de [80-120] mmHg.

En conclusión, la investigación permitió desarrollar un modelo y un método que permite la evaluación de variables como velocidad, presión, tracción esfuerzo y desplazamiento en un problema de dominios acoplados (fluido estructura) en dos vías, utilizando esquemas de solución como el modelo upwind, funciones de base radial y el método de elementos de frontera con números de Reynolds mayores a los reportados para el método.

\section{REFERENCIAS}

[1]. C. J, F. E, and B. L., Blood vessel replacement: 50 years of development and tissue engineering paradigms in vascular surgery, Physiological Research, 2009.

[2]. Y. I, H. J, et al., Desing of polycaprolactone vascular graft, Journal of Industrial Textiles, 2014.

[3]. M. M, S. M, et al., Estudio comparativo del uso de prótesis vasculares de ptfe a nivel de antebravo vs catéter permanente, Nefrología, 2006

[4]. S. Zeeshan, R. Jay, et al., Tissue engineering of acellular grafts capable of somatic growth in young lambs, Nature communications, 2016.
[5]. S. Henryk and G. Sean., The mechanical behavior of vascular grafts: A review, 2001.

[6]. B. Martina, C. Suncica, et al., Fluid-structure interaction in blood flow capturing non-zero longitudinal structure displacement, Journal of Computational Physics., no. 235, 2013.

[7]. C. Vincent, P. Goncalo, et al., High-order fluid-structure interaction in $2 \mathrm{~d}$ and $3 \mathrm{~d}$ application to blood flow in arteries, Journal of Computational and Applied Mathematics, 2013.

[8]. W. C and C. L, A small diameter elastic blood vessel wall prepared under pulsatile conditions from polyglycolic acid mesh and smooth muscle cells dfferentiated from adipose-derived stem cells., Biomaterials, vol. na, pp. 621_630, 2010.

[9]. B. Jhon, E. Claudia, and V. Javier., Evaluación hidromecánica de venasvyugulares bovinas frescas y fijadas en glutaraldehído para uso como bioimplante cardiovascular, Revista Colombiana de Cardiología, vol. 14, pp. 238_246, 2007.

[10]. D. Álamo J, C. Marsden, et al., Recent advances in the application of computational mechanics to the diagnosis and treatment of cardiovascular disease., Cardiovascular Translation Medicine, vol. na, pp. 781_805, 2009.

[11]. H. M. Chen and B. Yuri, Blood vessel tissues prestress modeling for vascular fluid-structure interaction simulation, Finite Elements in Analysisand Desing, 2011.

[12]. T. Y, Computational biomechanical studies of the human cardiovascular system., Journal of Biomechanics, vol. 40, p. na, 2007.

[13]. O. Pelliccioni and M. Cerrolaza, Análisis bidimensional de la interacción fluido-estructura en válvulas mecánicas de corazón utilizando técnicas de células autómatas., Revista de la facultad de Ingeniería universidad central de Venezuela., vol. na, p. na, 2006.

[14]. I. Yohsuke, O. Toshihiro, et al., Numerical methods for simulating blood flow at macro, micro, and multi scales,Journal of Biomechanics, 2015.

[15]. J. Joao, M. Alexandra, et al., A 3d non- newtonian fluidstructure interaction model for blood flow in arteries, Journal of Computational and applied Mathematics, 2010.

[16]. F. C. Baek, S. Taylor, et al., A computational framework for fluidsolid- growth modeling in cardiovascular modelation., Comput.Methods Appl.Mech.Engnr, vol. na, pp. 3583 3602., 2009.

[17]. C. P. F. J., Simulación del flujo sanguineo y su interacción con la pared arterial mediante modelo de elementos finitos. PhD thesis, Madrid, 2006.

[18]. Q. Alfio and F. Lucca, Mathemathical modeling and numerical simulation of the cardiovascular system.vJanuary 2002.

[19]. P. G, Pulsatile blood flow in a pipe., Computers \& Fluids, vol. 27, vpp. 367-380, 1998.

[20]. C. Felippa, Y. Park, and Others, Partitioned analysis of coupled mechanical systems, 2001.

[21]. F. L and V. A, Biological Fluid Dynamics. One Dimensional Models for blood Flow in The Human Vascular System., 2003.

[22]. U. S, C. D. M, et al., _Análisis computacional de flujos sanguineos utilizando modelos $3 \mathrm{~d}$ axisimétricos y el método de elementos finitos., Master's thesis, Uner, 2007.

[23]. B. Martin, Radial basis functions. Cambridge University Press. 
[24]. S. Y and C. G, _Local radial basis function based gridfree scheme for unsteady incompressible viscous flows, Journal of computational physics, vol. 227, pp. 8922_8948, 2008.

[25]. J. Talts, R. Raamat, and K. Jagomagi, Influence of pulse pressure variation on the results of local arterial compliance measurement: A computer simulation study, Computers in Biology and Medicine, vol. 39, pp. 707-712, 2009.

[26]. B. Abdessalem, B. Durand, et al., Fluid structure interaction in a free end textile vascular prosthesis., The European Physical Journal Applied Physics 31, pp. 211-216, 2005.

[27]. G. Mauricio, Boundary integral method for two phase systems with non-Newtonian dispersed phase. PhD thesis, Universidad Ponti_cia Bolivariana, 2007.

[28]. P. H and P. P, The use of stoke.s fundamental solution for the boundary only element formulation of the three dimensional navier stokes equations for moderate reynolds numbers, International journal for numerical methods in engieneering vol 37, 1994.

[29]. G. Sevin, The dual reciprocity boundary element method solution of fluid flow problems. PhD thesis, School of applied mathematics, 2010.

[30].F. WF and H. Power, Drm multidomain mass conservative interpolation appproach for the bem solution of the twodimensional navier-stokes equations, Computers and mathematics with applications 43, 2002.

[31]. L. OA, The Mathematical Theory of Viscous Incompressible Flow. Gordon and Breach, 1963.

[32]. Y. Jeon, _Hybridized supg and upwind numerical schemes for convection dominated diffusion problems, 2015.

[33]. B. C.A, Power, H. Florez, et al., A global meshless collocation particular solution method for solving the two-dimensional navier-stokes system of equations, Computer and mechanics with applications 65, 2013.

[34]. C. Felippa and T. Geers, Partitoned analysis for coupled mechanical systems vol 5. 1988.

[35]. J. Youngmok, Hybridied supg and upwind numerical schemes for convection dominated diffusion problems, Journal of Computational an, 2015. 